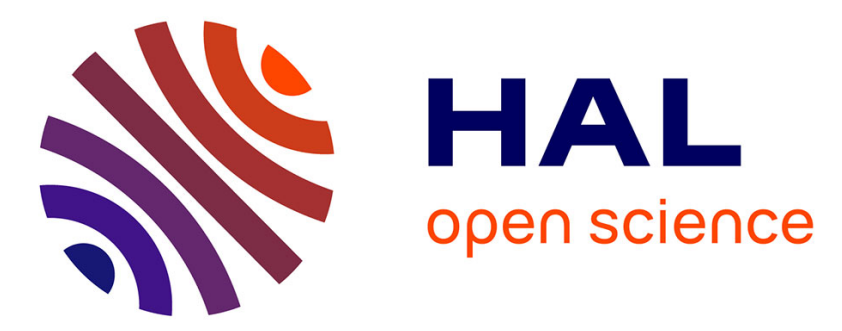

\title{
Optical indices and transport scattering coefficient of pyrolytic boron nitride: a natural thermal barrier coating for solar shields
}

Domingos de Sousa Meneses, Marianne Balat-Pichelin, Olivier Rozenbaum, Leire del Campo, Patrick Echegut

\section{To cite this version:}

Domingos de Sousa Meneses, Marianne Balat-Pichelin, Olivier Rozenbaum, Leire del Campo, Patrick Echegut. Optical indices and transport scattering coefficient of pyrolytic boron nitride: a natural thermal barrier coating for solar shields. Journal of Materials Science, 2016, 51 (9), pp.4660-4669. 10.1007/s10853-016-9781-2 . insu-01280535

\section{HAL Id: insu-01280535 \\ https://hal-insu.archives-ouvertes.fr/insu-01280535}

Submitted on 4 Mar 2016

HAL is a multi-disciplinary open access archive for the deposit and dissemination of scientific research documents, whether they are published or not. The documents may come from teaching and research institutions in France or abroad, or from public or private research centers.
L'archive ouverte pluridisciplinaire HAL, est destinée au dépôt et à la diffusion de documents scientifiques de niveau recherche, publiés ou non, émanant des établissements d'enseignement et de recherche français ou étrangers, des laboratoires publics ou privés. 


\title{
Optical indices and transport scattering coefficient of pyrolytic boron
}

\section{nitride: a natural thermal barrier coating for solar shields}

Domingos De Sousa Meneses ${ }^{\mathrm{a},{ }^{*}}$, Marianne Balat-Pichelin ${ }^{\mathrm{b}}$, Olivier Rozenbaum ${ }^{\mathrm{c}}$, Leire del Campo $^{\mathrm{a}}$, Patrick Echegut ${ }^{\mathrm{a}}$

${ }^{\text {a }}$ CNRS, CEMHTI UPR3079, Univ. Orléans, F-45071 Orléans, France

${ }^{\mathrm{b}}$ Laboratoire Procédés, Matériaux et Energie Solaire, PROMES-CNRS, 7 rue du Four Solaire, 66120 Font-Romeu Odeillo, France

${ }^{\mathrm{c}}$ ISTO, UMR 7327, 1 A rue de la Férollerie, 45100 Orléans, France

* Corresponding author.

Tel.:+33 238255534 Fax : +33238638103

E-mail address: desousa@cnrs-orleans.fr

Postal address : CEMHTI - UPR3079 CNRS Site Haute Température CS 90055, 1D avenue de la Recherche Scientifique, 45071 Orléans Cedex 2 France

\begin{abstract}
Absorption and scattering properties of pyrolytic boron nitride $(\mathrm{pBN})$ have been characterized by infrared spectroscopy. The strong dielectric anisotropy predicted by first principles calculations is confirmed by measurements performed on a highly oriented pBN sample. Optical properties of textured samples elaborated by chemical vapor deposition were identified from normal hemispherical reflectance and transmittance spectra by applying modified two flux and four flux transport models. It is also shown that coating carbon-carbon composites used to build solar shields with a pBN layer having an optimal thickness could improve the protection performance.
\end{abstract}


Keywords : Boron nitride; Dielectrics; Optical spectroscopy; Thermal barrier coating; Modelling

\section{Introduction}

The availability of accurate optical and thermal radiative properties of scattering materials is becoming strategic in laboratories and even more patently in the industrial sector. This knowledge is essential in material selection, for sizing solar absorbers [1] and thermal barrier coatings [2,3], to design and shape thermal shields which protect instrumentation and communication devices in solar probes such as PHOIBOS and Solar Probe Plus [4,5]. Extracting optical indices from experimental data acquired on single crystals is a well-known procedure [6], but doing the same task on heterogeneous samples still represents a difficult challenge. The full analysis of the problem needs a thorough experimental characterization of the surface and volumetric textures of materials and the use of computer intensive numerical methods for its resolution [7]. To bypass this difficulty, approximate analytical expressions for the identification of the absorption and scattering coefficients of complex media have been proposed. Models with increasing complexity going from the classic Kubelka-Munk approximation to multi-flux models have been reported in the literature. The comparison between exact numerical results and model predictions [8,9] and the analysis of absorbing and scattering coefficients retrieved with different models [10] allowed to bring out important conclusions on their accuracy and conditions of use. Among the models used in the characterization of ceramics, the modified two-flux [11] and the four-flux approximations [12] are very appealing since they combine easiness of use and good performance in the identification of absorbing and scattering properties. These models are used in this paper to analyze the semi-transparent spectral range of hexagonal pyrolytic boron nitride (pBN). This dielectric material has excellent thermal and chemical stability in high vacuum, low thermal 
conductivity in the axial direction, good resistance to thermal shock and is among the few ones that can suffer extreme conditions of temperature [4]. Due to its optical selectiveness (good infrared emitter and good reflector in the visible range), textured $\mathrm{pBN}$ was identified to be an appropriate candidate to coat heat shields of solar probes [5]. A thorough characterization of its optical properties is thus a necessary step to enable its use in thermal protection systems. The study of optical functions of boron nitride in crystalline forms by experimental means started a long time ago [13-16] and later by ab initio calculations [17-22]. In the case of hexagonal $\mathrm{BN}$ (the polymorph that matters here), the collection of the data published in the literature for the complex refractive index shows that it is rather well characterized for a polarization in the $a b$ plane; on the contrary values along the $c$ axis are not well established. Additionally, the published data on the optical properties in the infrared range of textured pBN elaborated by chemical vapor deposition (CVD) are scarce [23,24]. In this paper, we report diffuse reflectance and transmittance spectra acquired on such a pBN material. The data are analyzed within a theoretical framework that allows retrieving the spectral dependencies of the refractive index, the absorption coefficient and the scattering coefficient from normal hemispherical reflectance and transmittance measurements. The results are compared and discussed in light of the data already published in the literature and a study of the impact of the thickness of $\mathrm{pBN}$ coatings on the performance of thermal shields equipping solar probes is also proposed.

\section{Experimental section}

\subsection{Sample description}

The pyrolytic boron nitride ( $\mathrm{pBN}$ ), provided by Ceramisis (UK), was elaborated by using the CVD technique. The layer deposited onto machined graphite mandrels at temperatures higher 
than $2100 \mathrm{~K}$, is separated upon cooling from the mandrels to form a free-standing bloc that is machined to the customer specification. The disk-shaped samples used in this work have a high purity (>99.995\%) and a diameter of $40 \mathrm{~mm}$ (see reference [4] for a more complete description of the material properties). A highly oriented pyrolytic hexagonal boron nitride homogeneous sample (hopBN) having a mosaic spread lower than $2^{\circ}$ was also investigated as a reference. The sample, obtained by compression annealing at high temperature, has a plate shape $\left(10 \times 10 \times 0.4 \mathrm{~mm}^{3}\right)$ and comes from the Carbon Products Division of the Union Carbide Corporation [25].

\subsection{Microstructural characterization}

The pBN samples have a density of $(2.10 \pm 0.02) \mathrm{g} \mathrm{cm}^{-3}$ which corresponds to a total porosity of about $8 \%$. The estimation was made on a cylindrical sample by evaluating the ratio of the total mass and volume determined geometrically from known dimensions (40 mm diameter and $2 \mathrm{~mm}$ thick). A X-ray diffraction pattern was acquired between 10 and $100^{\circ}(2 \theta)$ at room temperature with a $0.0164^{\circ}$ step size on a D8 Advance Bruker Bragg-Brentano diffractometer (CuK $\alpha$ radiation) equipped with a Vantec-1 linear detector (Fig.1).

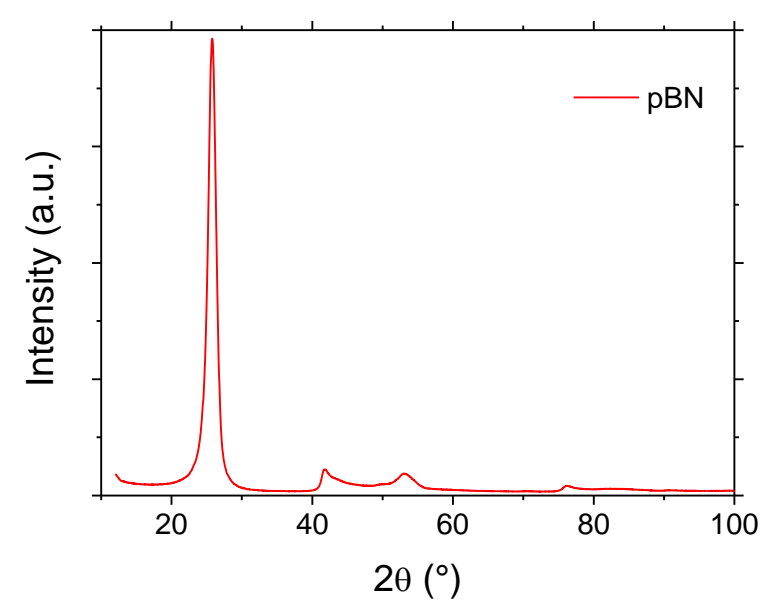

Fig. 1: X-ray diffraction pattern of $\mathrm{pBN}$. 
The determination of the lattice parameters was done by applying the LeBail method which leaded to $a=2.467(2)$ and $c=6.873(2)$. The analysis of the peaks appearing in the x-ray diffractogram of a bulk sample confirms that the material is made of a distribution of hexagonal boron nitride crystallites with orientational disorder and a mean size estimated to be around $15 \mathrm{~nm}$.

An attempt to probe the spatial and size distributions of the pores was realized by 3D image analysis. Microtomography analyses were performed using a laboratory-based CT-scanner (Nanotom 180NF; GE Phoenix|X-ray, Wunstorf, Germany). In order to obtain low penetrating X-ray a target made of molybdenum was selected and an operating voltage of 80 $\mathrm{kV}$ with a filament current of $180 \mu \mathrm{A}$ was applied. The distance between the $\mathrm{X}$-ray source and the sample and between the X-ray source and the detector was 5.5 and $500 \mathrm{~mm}$, respectively, giving a voxel size of $1.1 \mu \mathrm{m}$ and, therefore, a resolution around $3 \mu \mathrm{m}$. For this resolution, the pBN 3D image (not shown here) is not able to distinguish the pores and the image can be considered as homogeneous. This indicates that the characteristic size of the volumetric texture of the material is lower than $3 \mu \mathrm{m}$ which is close to the best resolution achievable with this experimental setup.

SEM imaging was carried out with a Hitachi S-4500 and surface roughness was probed with a Leica DCM-3D. Fig. 2 shows the surface morphology of pBN sample at two magnification where one can see some holes and big grains leading to some roughness. 

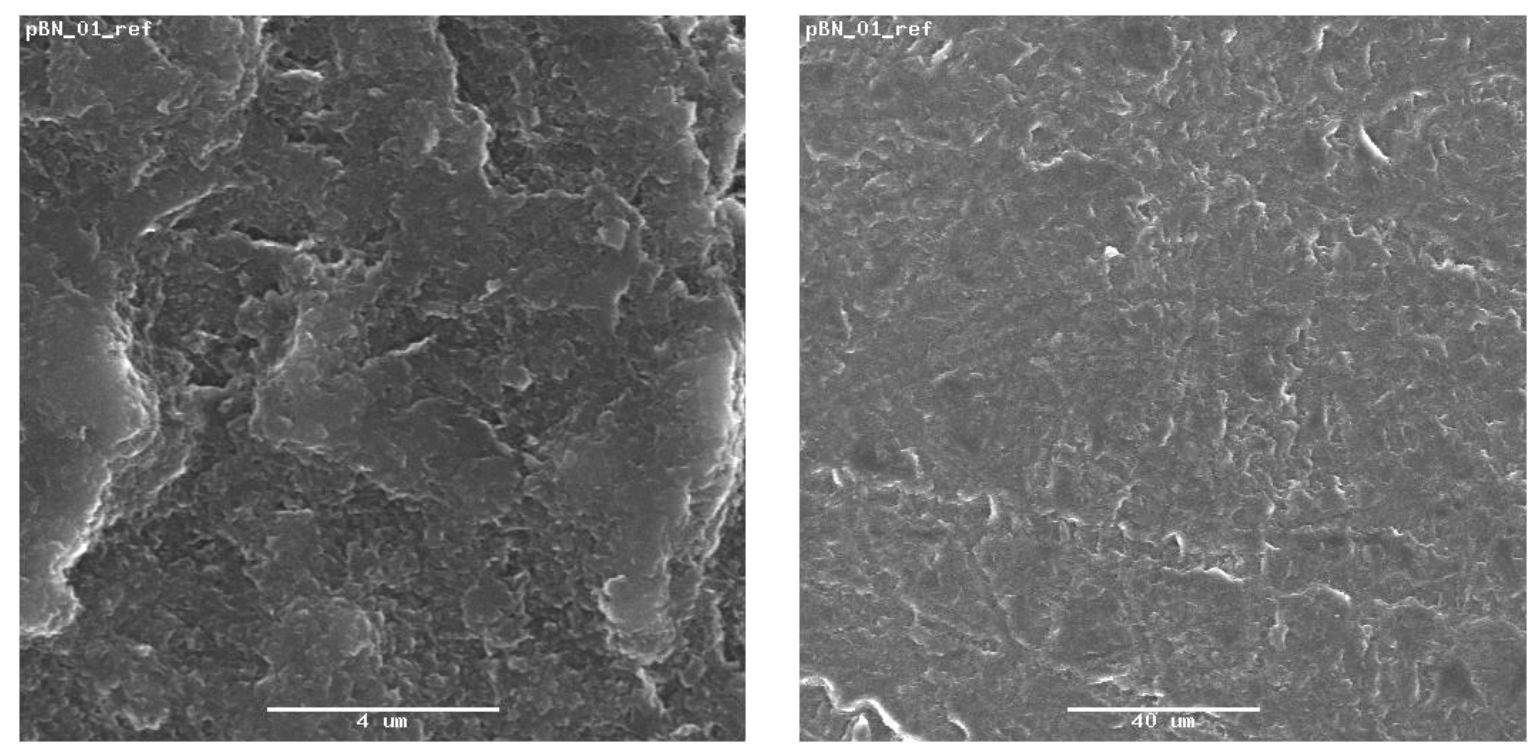

Fig. 2: SEM images of the pBN surface, scale bars: on the left $4 \mu \mathrm{m}$ and on the right $40 \mu \mathrm{m}$

The roughness parameters Sa (arithmetic), Sq (quadratic, RMS) and Sz (max height) are respectively equal to $1.04,1.39$ and $11.65 \mu \mathrm{m}$ as some traces of polishing are still visible on the images of Fig. 3. Looking at these 3D images, it can be seen that this roughness will act on the optical measurements presented here after. 

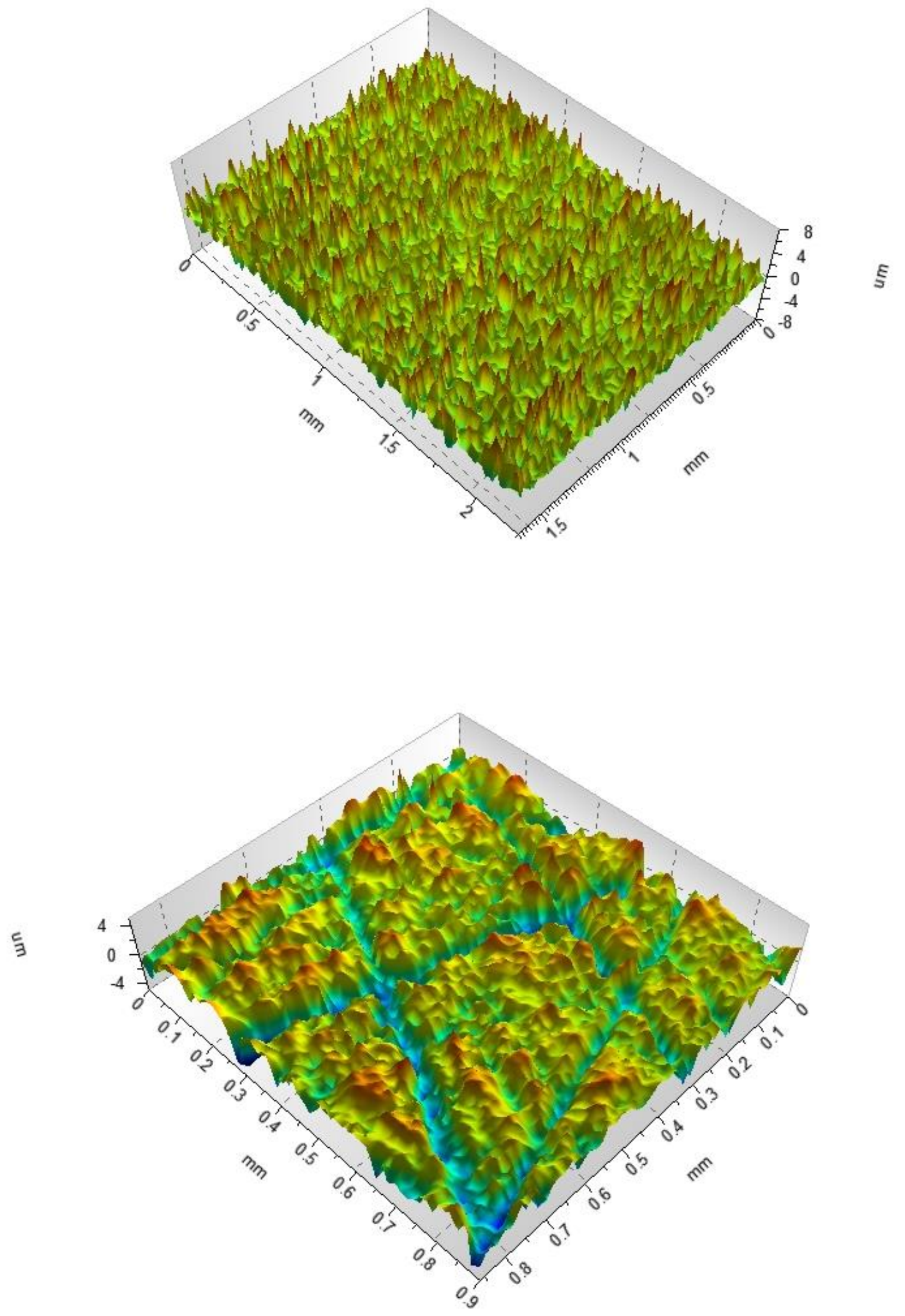
Fig. 3: 3D images with different magnifications (top : x10, bottom : x20) of as-received pBN obtained by 3D profilomtery

\subsection{Optical measurements}

A set of normal-hemispherical reflectance and transmittance spectra has been acquired on pBN samples with increasing thicknesses $\left(1.5\right.$ to $6 \mathrm{~mm}$ ) from 500 to $10000 \mathrm{~cm}^{-1}$ with a Bruker Vertex 70 spectrometer at room temperature (Fig. 4). The sample compartment was equipped with a 6 inch integrating sphere covered with an infragold coating [26]. Quartz halogen lamp and globar sources were used with a $\mathrm{Ge} / \mathrm{KBr}$ beam splitter and a Mercury Cadmium Telluride (MCT) detector to probe Near InfrarRed (NIR) and Mid InfraRed (MIR), respectively.
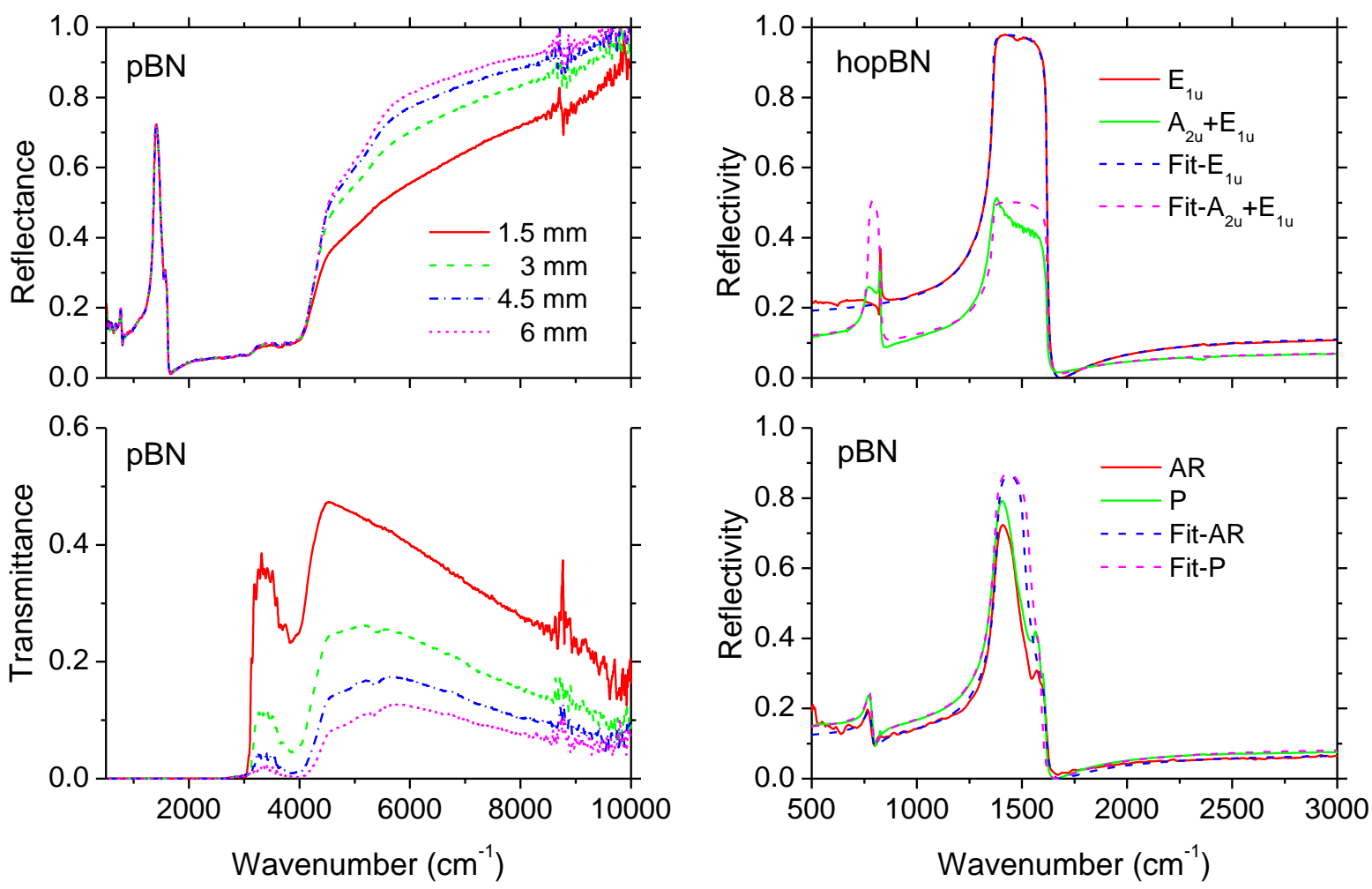
Fig. 4: Normal hemispherical reflectance (left-top) and normal hemispherical transmittance spectra (left-bottom) of pBN-AR for several sample thicknesses going from $1.5 \mathrm{~mm}$ to $6 \mathrm{~mm}$. (right-top) Non polarized near normal reflectivity spectra measured on a z-cut surface of hopBN $\left(\perp c, E_{1 u}\right)$ with fits using (Eq.4) and on a x-cut surface $\left(A_{2 u}+E_{1 u}\right)$. (right-bottom) Near normal reflectivity spectra with fits using (Eq.4) of as-received (AR) and polished (P) pBN.

The measurement of specular reflectivity spectra at near normal incidence were performed with a Bruker Hyperion 3000 FTIR microscope coupled to a Bruker Vertex 80v spectrometer using a gold mirror reference. The microscope was equipped with a $15 \mathrm{X}$ Cassegrain objective. MIR data were obtained with a $\mathrm{Ge} / \mathrm{KBr}$ beam splitter and a MCT detector. All the spectra were recorded with an instrumental resolution of $4 \mathrm{~cm}^{-1}$.

\section{Modeling of the optical response}

\subsection{Opacity domain}

The symmetry of the crystal structure of hexagonal $\mathrm{BN}$ is given by its space group $P 6_{3} /$ $m m c\left(D_{6 h}^{4}-194\right)$. The primitive unit cell contains two formula units with B and $\mathrm{N}$ atoms occupying Wyckoff positions $(2 c)$ and $(2 d)$, respectively [27]. With this knowledge, the reducible representation $\Gamma_{v i b}$ of the optical modes at the center of Brillouin zone is determined by applying group theory:

$$
\Gamma_{v i b}=A_{2 u}(I R)+2 B_{1 g}+E_{1 u}(I R)+2 E_{2 g}(\text { Raman })
$$

Among the irreducible representations included in the decomposition, only $A_{2 u}$ and $E_{1 u}$ are IR active. The existence of a single optical mode with $A_{2 u}$ symmetry and a degenerate one 
with $E_{1 u}$ symmetry leads to dielectric functions $\varepsilon_{\|, \perp}$ (for parallel $(\| c)$ and perpendicular $(\perp c)$ polarizations) including both a single phonon term. Selecting a Lorentz model with three parameters to take account for the susceptibility of the oscillators, the physical expressions for the dielectric functions reduce to:

$$
\varepsilon_{\|, \perp}(\omega)=\varepsilon_{\|, \perp}^{\infty}+\frac{\omega_{\|, \perp}^{2} \Delta \varepsilon_{\|, \perp}}{\omega_{\|, \perp}^{2}-\omega^{2}-i \gamma_{\|, \perp}}
$$

$\Delta \varepsilon_{j}, \omega_{j}$ and $\gamma_{j}$ represent the dielectric contribution, the wavenumber and the damping of the phonon $(j \rightarrow \|, \perp)$, respectvely. $\varepsilon_{j}^{\infty}$ is the high frequency electronic contribution. These dielectric function models are used in the following to take account of the vibrational response of the homogeneous sample (hopBN) but they are not directly applicable in such a form to characterize textured $\mathrm{pBN}$ samples.

In the phonon range (between 600 and $2000 \mathrm{~cm}^{-1}$ ), the typical dimensions of roughness and porosity of $\mathrm{pBN}$ are less than the wavelengths of infrared radiation (see Fig. 3). This enables the use of homogenization procedures to deduce effective dielectric properties. By assuming a random distribution of the orientations of the crystallites within $\mathrm{pBN}$, an average dielectric function $\varepsilon_{h}$ for the $\mathrm{pBN}$ matrix can be obtained by applying:

$$
\varepsilon_{h}(\omega)=\frac{1}{3} \varepsilon_{\|}(\omega)+\frac{2}{3} \varepsilon_{\perp}(\omega)
$$

Then taking into account the roughness and porosity at and near sample surface by using the Bruggeman mixing formula [28] :

$$
f \frac{\varepsilon_{e}-1}{2 \varepsilon_{e}+1}+(1-f) \frac{\varepsilon_{e}-\varepsilon_{h}}{2 \varepsilon_{e}+\varepsilon_{h}}=0
$$

the effective dielectric function $\varepsilon_{e}$ of the $\mathrm{pBN}$ material is obtained by computing $\varepsilon_{e}=$ $\frac{1}{4}\left(\gamma+\sqrt{\gamma^{2}+8 \varepsilon_{h}}\right)$ with $\gamma=(3 f-1)+(2 f-3) \varepsilon_{h}$.

The reflectivity at near normal incidence is obtained by applying the Fresnel formula: 


$$
R(\omega)=\left|\frac{\sqrt{\varepsilon(\omega)}-1}{\sqrt{\varepsilon(\omega)}+1}\right|^{2}
$$

A first possibility to retrieve the dielectric properties of boron nitride compounds in the opacity domain is to fit their reflectivity spectra with Eq. (4) and the dielectric function models $\varepsilon$ introduced herein before. The complex refractive index $\tilde{n}$ is then evaluated by using: $\tilde{n}(\omega)=n(\omega)+i k(\omega)=\sqrt{\varepsilon(\omega)}$. In the later expression, $n$ is the index of refraction and $k$ stands for the absorption index which is directly linked to the absorption coefficient by the following relation: $K(\omega)=$ $4 \pi k(\omega) \omega$.

Another way to obtain the information is to compute the phase spectrum $\theta$ from the reflectivity spectrum $R$ by using one of the Kramers-Kronig relations:

$$
\theta(\omega)=-\frac{1}{2 \pi} P \int_{0}^{\infty} \ln \left|\frac{s+\omega}{s-\omega}\right| \frac{d \ln [R(s)]}{d s} d s
$$

The knowledge of $R$ and $\theta$ is enough to deduce the complex refractive index of the material [6].

\subsection{Semi-transparency domain}

In the semi-transparent domain of $\mathrm{pBN}$, above $3000 \mathrm{~cm}^{-1}$, light scattering rapidly strengthens with increasing wavenumber. Effective medium theories do not apply anymore. So the analysis of the normal hemispherical reflectance and transmittance spectra was made by applying two multi-flux models. The modified two flux approximation (M2FA) has proven to be applicable to many practical problems [11]. The associated analytical expressions for the reflectance $R_{d}$ and the transmittance $T_{d}$ of the textured material have only three adjustable parameters: the index of refraction $n$, the absorption coefficient $K$ and the transport scattering 
coefficient $\sigma_{t r}$. In the case of a sample with a parallel plate geometry of thickness $L$, these quantities are given by:

$$
\begin{gathered}
R_{d}^{M 2 F A}=\frac{\left(1-R_{s}\right)^{2}}{1-R_{s}^{2} E_{t r}^{2}}\left[\frac{R_{s}}{\left(1-R_{s}\right)^{2}}+R_{s} E_{t r}^{2}+D_{t r}\left(1+\chi \frac{k_{1} E_{t r}+k_{2} \varphi s+k_{2} c}{s+\varphi^{2} s+2 \varphi c}+R_{s} E_{t r}^{2}\right)\right] \\
T_{d}^{M 2 F A}=\frac{\left(1-R_{s}\right)^{2}}{1-R_{s}^{2} E_{t r}^{2}}\left[E_{t r}+D_{t r}\left(\chi \frac{k_{1} \varphi s E_{t r}+k_{1} c E_{t r}+k_{2}}{s+\varphi^{2} s+2 \varphi c}+\left(1+R_{s}\right) E_{t r}\right)\right]
\end{gathered}
$$

In Eqs. (6) and (7), the symbols have the following definitions: $\omega_{t r}=\frac{\sigma_{t r}}{K+\sigma_{t r}}, \mu=\sqrt{1-\frac{1}{n^{2}}}$,

$$
\begin{aligned}
& \chi=\frac{2}{1+\mu} \sqrt{\frac{1-\omega_{t r}}{1-\mu \omega_{t r}}}, R_{s}=\left(\frac{n-1}{n+1}\right)^{2}, D_{t r}=\frac{\chi^{2} \sigma_{t r}}{2 n^{2} K\left(1+R_{s}\right)\left(\chi^{2}-1\right)}, \tau_{t r}=\left(K+\sigma_{t r}\right) L, E_{t r}=\exp \left(-\tau_{t r}\right), \\
& c=\cosh \left(\chi \tau_{t r}\right), s=\sinh \left(\chi \tau_{t r}\right), \varphi=\frac{2\left(1-R_{s}\right)}{\chi\left(1+R_{s}\right)(1+\mu)}, k_{1}=\left(\chi^{-1}-\varphi\right)-\left(\chi^{-1}+\varphi\right) R_{s}, \\
& k_{2}=\left(\chi^{-1}-\varphi\right) R_{s} E_{t r}^{2}-\left(\chi^{-1}+\varphi\right) .
\end{aligned}
$$

The second model selected to analyze the pBN spectra is the four flux approximation (4FA) recently used to investigate the infrared response of yttria-stabilized zirconia thermal barrier coatings $[3,12]$. The associated analytical expressions for reflectance and transmittance for a parallel plate of thickness $L$ also include three adjustable parameters: the index of refraction $n$, the absorption coefficient $K$ and the scattering coefficient $\sigma$ :

$$
\begin{gathered}
R_{d}^{4 F A}=R_{s}+C_{1}\left[\left(1-R_{s}\right) C_{2}+A\left(2+6 C_{2}+\frac{(1-\beta) C_{3}+(1+\beta) C_{4}}{\eta_{1} \sinh (2 \beta \tau)+\eta_{2} \cosh (2 \beta \tau)}\right)\right] \\
T_{d}^{4 F A}=C_{1}\left[\left(1-R_{s}\right) e^{-\tau}+A\left(3 e^{-\tau}+C_{2} e^{\tau}+\frac{(1+\beta) C_{3} e^{-2 \beta \tau}+(1-\beta) C_{4} e^{2 \beta \tau}}{\eta_{1} \sinh (2 \beta \tau)+\eta_{2} \cosh (2 \beta \tau)}\right)\right]
\end{gathered}
$$

Where $\tau=(K+\sigma) L, \beta=\sqrt{\frac{K}{K+\sigma}}, A=\frac{\sigma\left(1-R_{i}\right)}{12 K-4 \sigma}, C_{1}=\frac{1-R_{S}}{1-R_{S}^{2} e^{-2 \tau}}, C_{2}=R_{S} e^{-2 \tau}, C_{3}=$ $\lambda_{1} \mu_{1} e^{2 \beta \tau}-\lambda_{2} \mu_{2} e^{-\tau}, C_{4}=\lambda_{1} \mu_{2} e^{-\tau}-\lambda_{2} \mu_{1} e^{-2 \beta \tau}$ and $\lambda_{1}=(1+\beta)-R_{i}(1-\beta), \lambda_{2}=$ $(1-\beta)-R_{i}(1+\beta), \mu_{1}=\left(R_{i}-3\right)-R_{s}\left(3 R_{i}-1\right) e^{-2 \tau}, \mu_{2}=3\left(R_{i}-R_{s}\right)-\left(1-R_{S} R_{i}\right)$, 


$$
\begin{aligned}
& \eta_{1}=\left(1-R_{i}\right)^{2}+\beta^{2}\left(1+R_{i}\right)^{2}, \eta_{2}=2 \beta\left(1-R_{i}^{2}\right), R_{i}=1-\frac{1}{n^{2}}\left[\frac{1}{2}-\frac{(3 n+1)(n-1)}{6(n+1)^{2}}-\right. \\
& \left.\frac{n^{2}\left(n^{2}-1\right)^{2}}{\left(n^{2}+1\right)^{3}} \ln \left(\frac{n-1}{n+1}\right)+\frac{2 n^{3}\left(n^{2}+2 n-1\right)}{\left(n^{2}+1\right)\left(n^{4}-1\right)}-\frac{8 n^{4}\left(n^{4}+1\right)}{\left(n^{2}+1\right)\left(n^{4}-1\right)^{2}} \ln (n)\right] .
\end{aligned}
$$

\section{Results and discussion}

\subsection{Lattice dynamics}

The retrieval of the dielectric functions of hopBN and $\mathrm{pBN}$ within the phonon range have been made by fitting the experimental reflectivity spectra with Eq. (4) and the adequate dielectric function model, i.e. Eq. (1) for hopBN and Eq. (3) for pBN-AR (As-Received pBN) and pBN-P (Polished pBN). The extracted data reported in Fig. 5 for $\mathrm{pBN}$ are compared to the results obtained by applying also numerical inversion based on the Kramers-Kronig relation (Eq. 5). As shown in the figure, there is a very good overall agreement between the data evaluated by the two procedures. The only exception is the spectral range $\left[1370,1620 \mathrm{~cm}^{-1}\right]$ between the transverse and the longitudinal optic wavenumbers of the stronger IR active phonon of $\mathrm{pBN}$. This discrepancy was awaited and is explained by the inability of the oversimplified Bruggeman effective medium theory to quantitatively describe the complex optical response of the textured material within this spectral range. Indeed, in this specific zone near the phonon resonance, the strong dispersion of the dielectric function associated to heterogeneities such as roughness and porosity close to the interface, activate surfaces modes that affect the reflectivity of the material [29]. Away from the resonance the results obtained within this framework are consistent with those coming from the Kramers-Kronig inversion. Between the transverse and longitudinal optic wavenumbers of the high frequency phonon, the Kramers-Kronig inversion gives a better estimation of the dielectric function of the material. 

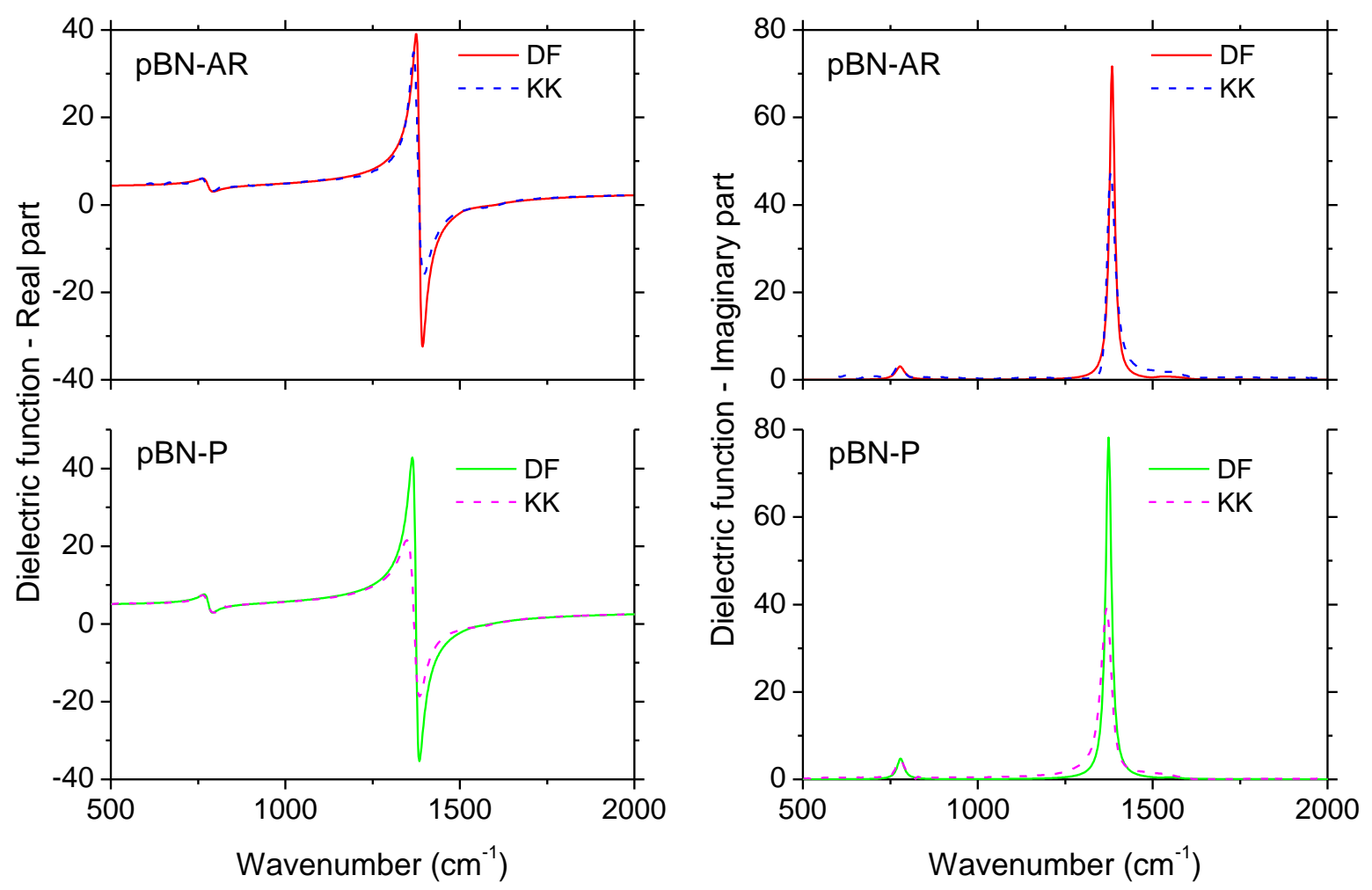

Fig. 5: Real and imaginary parts of the dielectric functions of as-received (AR) and polished (P) pBN. DF represents values obtained by fitting the experimental spectra with a dielectric function model, KK are the ones extracted by applying Kramers-Kronig relations.

The model parameters associated to the lattice dynamics are reported in Table 1 and Table 2.

Table 1. Dielectric contributions, wavenumbers and dampings in $\mathrm{cm}^{-1}$ of $A_{2 u}(\|)$ and $E_{1 u}(\perp)$ IR active phonons for different types of hexagonal BN. Data associated to hopBN, pBN-P and pBN-AR are parameters of Eq. (1) resulting from the fit of the reflectance spectra reported in Fig. 4. Experimental and first principles values reported in the literature are also given for comparison.

\begin{tabular}{|l|c|c|c|c|c|c|c|c|c|}
\hline Material & $\omega_{\|}(T O)$ & $\omega_{\|}(L O)$ & $\gamma_{\|}$ & $\Delta \varepsilon_{\|}$ & $\omega_{\perp}(T O)$ & $\omega_{\perp}(L O)$ & $\gamma_{\perp}$ & $\Delta \varepsilon_{\perp}$ & Ref. \\
\hline
\end{tabular}




\begin{tabular}{|c|c|c|c|c|c|c|c|c|c|}
\hline hopBN & $770 \pm 3$ & $826 \pm 4$ & $7 \pm 4$ & $\begin{array}{l}0.3 \pm 0 . \\
04\end{array}$ & $1365 \pm 2$ & $1622 \pm 3$ & $4.5 \pm 2$ & $\begin{array}{l}1.82 \pm 0 . \\
05\end{array}$ & [*] \\
\hline pBN-P & $779 \pm 3$ & & $24 \pm 5$ & $\begin{array}{l}0.5 \pm 0 . \\
02\end{array}$ & $1374 \pm 3$ & & $20 \pm 4$ & $\begin{array}{l}1.94 \pm 0 . \\
05\end{array}$ & {$[*]$} \\
\hline $\mathrm{pBN}-\mathrm{AR}$ & $778 \pm 3$ & & $30 \pm 5$ & $\begin{array}{l}0.5 \pm 0 . \\
02\end{array}$ & $1384 \pm 3$ & & $18 \pm 4$ & $2 \pm 0.05$ & {$[*]$} \\
\hline $\begin{array}{l}\text { Polycrystall } \\
\text { ine sample }\end{array}$ & 783 & 828 & 8 & 0.53 & 1367 & 1610 & 29 & 1.87 & [13] \\
\hline CVD-pBN & $770 \pm 3$ & & $18 \pm 4$ & $\begin{array}{l}0.07 \pm \\
0.002\end{array}$ & $1383 \pm 5$ & & $30 \pm 4$ & $\begin{array}{l}0.39 \pm 0 . \\
005\end{array}$ & [14] \\
\hline $\begin{array}{l}\text { h-BN1 } \\
\text { h-BN2 }\end{array}$ & $\begin{array}{l}766 \\
773\end{array}$ & $\begin{array}{l}811 \\
820\end{array}$ & $\begin{array}{l}28 \\
60\end{array}$ & & $\begin{array}{l}1400 \\
1406\end{array}$ & $\begin{array}{l}1586 \\
1570\end{array}$ & $\begin{array}{l}143 \\
130\end{array}$ & & [15] \\
\hline $\begin{array}{l}\text { CVD Thin } \\
\text { Films }\end{array}$ & 790 & 828 & 30 & & 1386 & 1599 & 50 & & [16] \\
\hline DFT-LDA & 754 & 823 & & & 1382 & 1614 & & & [18] \\
\hline DFT-LDA & 745 & & & & 1375 & 1607 & & & [19] \\
\hline DFT-LDA & 757 & 783 & & & 1366 & 1587 & & & [20] \\
\hline $\begin{array}{l}\text { First } \\
\text { principles }\end{array}$ & 746 & 819 & & 0.61 & 1372 & 1610 & & 1.83 & [21] \\
\hline $\begin{array}{l}\text { DFT-LDA } \\
\text { DFT-GGA }\end{array}$ & $\begin{array}{l}760 \\
731\end{array}$ & $\begin{array}{l}824 \\
797\end{array}$ & & & $\begin{array}{l}1376 \\
1401\end{array}$ & $\begin{array}{l}1610 \\
1637\end{array}$ & & & [22] \\
\hline
\end{tabular}

[*] this work

Table 2. Static and high frequency dielectric constants of hexagonal BN samples of this work (hopBN, pBN-P and pBN-AR) and other ones reported in the literature. $p$ is the porosity involved in the formulation of the effective dielectric function (Eq. (3)) and $\langle\varepsilon\rangle$ represent an average non polarized value.

\begin{tabular}{|l|c|c|c|c|c|c|c|l|}
\hline Material & $\varepsilon_{\|}^{0}$ & $\varepsilon_{\|}^{\infty}$ & $\varepsilon_{\perp}^{0}$ & $\varepsilon_{\perp}^{\infty}$ & $p$ & $\left\langle\varepsilon^{0}\right\rangle$ & $\left\langle\varepsilon^{\infty}\right\rangle$ & Ref. \\
\hline hopBN & $2.3 \pm 0.1$ & $2 \pm 0.1$ & $6.3 \pm 0.1$ & $4.4 \pm 0.1$ & & & & {$[*]$} \\
\hline pBN-P & $3 \pm 0.1$ & $2.5 \pm 0.1$ & $6.4 \pm 0.1$ & $4.5 \pm 0.1$ & $\begin{array}{l}0.08 \pm \\
0.01\end{array}$ & $5.3 \pm 0.1$ & $3.8 \pm 0.1$ & {$[*]$} \\
& & & & & & \\
\hline
\end{tabular}




\begin{tabular}{|l|l|l|l|l|l|l|l|l|}
\hline pBN-AR & $3 \pm 0.1$ & $2.5 \pm 0.1$ & $6.5 \pm 0.1$ & $4.5 \pm 0.1$ & $0.15 \pm$ \\
0.02 & $4.2 \pm 0.1$ & $3.1 \pm 0.1$ & {$[*]$} \\
\hline $\begin{array}{l}\text { Polycrystalline } \\
\text { sample }\end{array}$ & 5.09 & 4.1 & 7.04 & 4.95 & & & & {$[13]$} \\
\hline CVD-pBN & & & & & & 3.55 & & {$[14]$} \\
\hline $\begin{array}{l}\text { h-BN1 } \\
\text { h-BN2 }\end{array}$ & & & & & & 3.2 & & {$[15]$} \\
\hline $\begin{array}{l}\text { CVC-Thin } \\
\text { films }\end{array}$ & & 2.72 & & 4.71 & & & & \\
\hline OLCAO & & 2.21 & & 4.32 & & & 3.61 & {$[17]$} \\
\hline DFT-LDA & 3.38 & 2.84 & 6.61 & 4.85 & & & & {$[18]$} \\
\hline First principles & 3.57 & 2.95 & 6.71 & 4.87 & & & & {$[21]$} \\
\hline
\end{tabular}

[*] this work

The parameters obtained in this work for the in plane $E_{1 u}(\perp)$ symmetry vibrations are in excellent agreement with experimental data found in the literature and values computed from first principles (see Table 1). The small dispersion of phonon wavenumbers observed for this symmetry is essentially due to differences in size of the crystalline domains present in textured samples. In a thorough study by Raman spectroscopy, Nemanich et al [30] evidenced a position shift towards higher wavenumbers and broadening of the $E_{2 g}$ mode $\left(1366 \mathrm{~cm}^{-1}\right)$ as the crystalline domains decrease in size. They observed a linear increase of parameter values versus reciprocal size of the crystallites. A similar trend is observed in our data for $A_{2 u}$ and $E_{1 u}$ phonons, the hopBN sample has narrower modes and the phonon resonances are located at lower wavenumbers as compared to those of the textured pBN sample. Instead, a comparison of experimental and first principle literature values obtained for static and high frequency dielectric constants for the $A_{2 u}$ (II) mode shows large deviations (Table 2). Such an observation led Ohba et al [18] and Cai et al [21] to question the validity of the experimental values published by Geick et al [13]. Our values indicate a high anisotropy of BN and are closer to values published for thin films [16] and those evaluated from first principles 
$[17,18,21]$. Concerning the analysis of the textured $\mathrm{pBN}$ samples, the total porosity extracted for the polished pBN-P sample (8\%) by using the Bruggeman effective medium approximation is identical to the one obtained through a density measurement. By contrast, the fit of the infrared response of the as-received sample (pBN-AR) leads to a total porosity of $15 \%$. This is not surprising since the surface roughness acts as an intermediate layer with higher porosity that diminishes the refractive index contrast at the air/sample interface.

\subsection{Absorption and scattering coefficients}

M2FA and 4FA models have been used to extract the absorption and scattering coefficients of pBN-AR samples having increasing thicknesses (Fig. 6). A least square fit procedure was applied on couples of normal hemispherical reflectance and transmittance spectra in the semitransparent spectral range (above $3000 \mathrm{~cm}^{-1}$ ). The absorption and scattering coefficients were the only adjustable parameters used in the optimization process; the spectral dependence of the index of refraction of $\mathrm{pBN}$ was imposed to be the continuation in the semi-transparent zone of the analytic solution obtained for $\mathrm{pBN}-\mathrm{AR}$ in the opacity domain. 

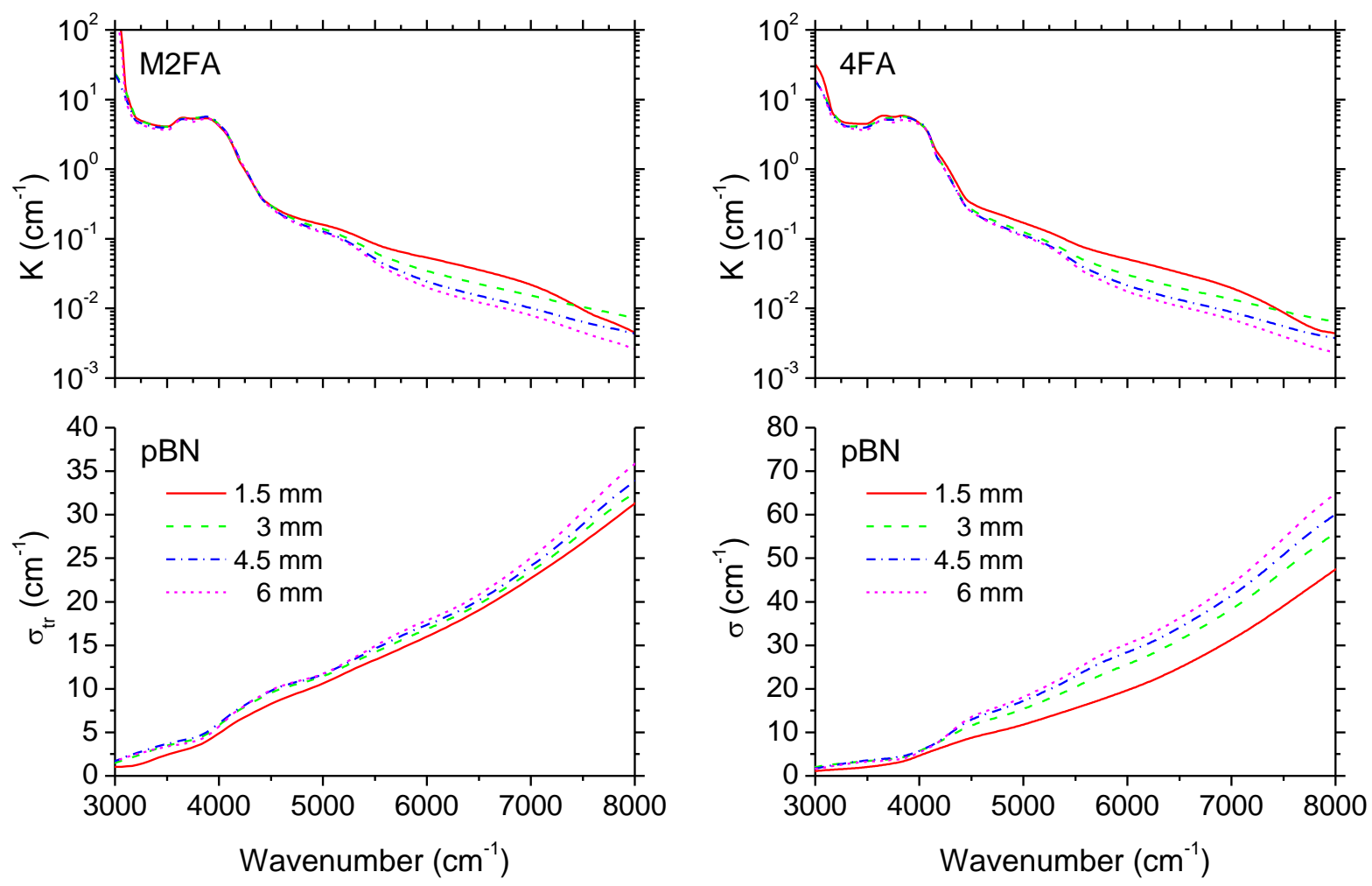

Fig. 6: Absorption and scattering coefficients of pBN-AR identified with M2FA and 4FA models for different sample thicknesses.

The values extracted for the absorption coefficient using both models are analogous, but going into details, some differences are nevertheless noticed. For values of absorption coefficient ranging from 0.1 to $10 \mathrm{~cm}^{-1}$, the M2FA model gives nearly identical values whatever the sample thickness investigated in this work which is the expected result. In the case of 4FA model, a small dispersion of the identified data is visible. This indicates that the M2FA model is better in the reproduction of light scattering in pBN-AR for this range of absorption coefficients. For weaker $K$ values, both approximations lead to data that present a deviation towards smaller values with increasing sample thickness. This evolution is concomitant with the opposite tendency observed for the scattering coefficient (it increases 
with thickness). Making the hypothesis that all the studied pBN-AR samples have identical impurity levels and same texture, one expects single spectral dependences for the absorption and scattering coefficients. Thus, a part of the observed thickness dependence is attributed to the inability of these models to fully describe the radiation scattering inside pBN-AR. The scattering functions used to obtain these analytical expressions are indeed simplified models that lead to an increased deviation with increasing scattering coefficient. The features appearing in the spectrum of the absorption coefficient and emerging from the global exponential decrease with increasing wavenumbers are associated to peaks in high order density of states corresponding to multi-phonon processes. The spectral trend observed for the scattering coefficient is power law like. Its exponent is close to 4 at low wavenumbers and then decreases slightly in the NIR range. This indicates that the size of most of the scatterers are well smaller than infrared wavelengths which is fully consistent with the texture analysis of pBN-AR. With increasing wavenumbers, radiation scattering changes continuously from Rayleigh like scattering towards an intermediate regime. In the measured spectral range the limit where scattering fully follows geometric optic laws is not visible. Below $5000 \mathrm{~cm}^{-1}$, the M2FA model leads to the same spectral dependence for the scattering coefficient whatever the sample thickness (if one discards the data obtained for the thinner sample). This confirms that this model is particularly good in the retrieval of the optical properties of $\mathrm{pBN}-\mathrm{AR}$ in the spectral range where the absorption dominates the scattering process.

\subsection{Solar probe shield application}

Due to their outstanding mechanical properties and resistance to high temperatures, carboncarbon composites (Fig. 7) are among the most interesting materials to design thermal shields for solar probe space missions [4,5]. Nonetheless and as shown in Fig. 7, their optical 
properties are far from being optimal for this type of application since they are poor infrared emitters and bad reflectors in the visible range.
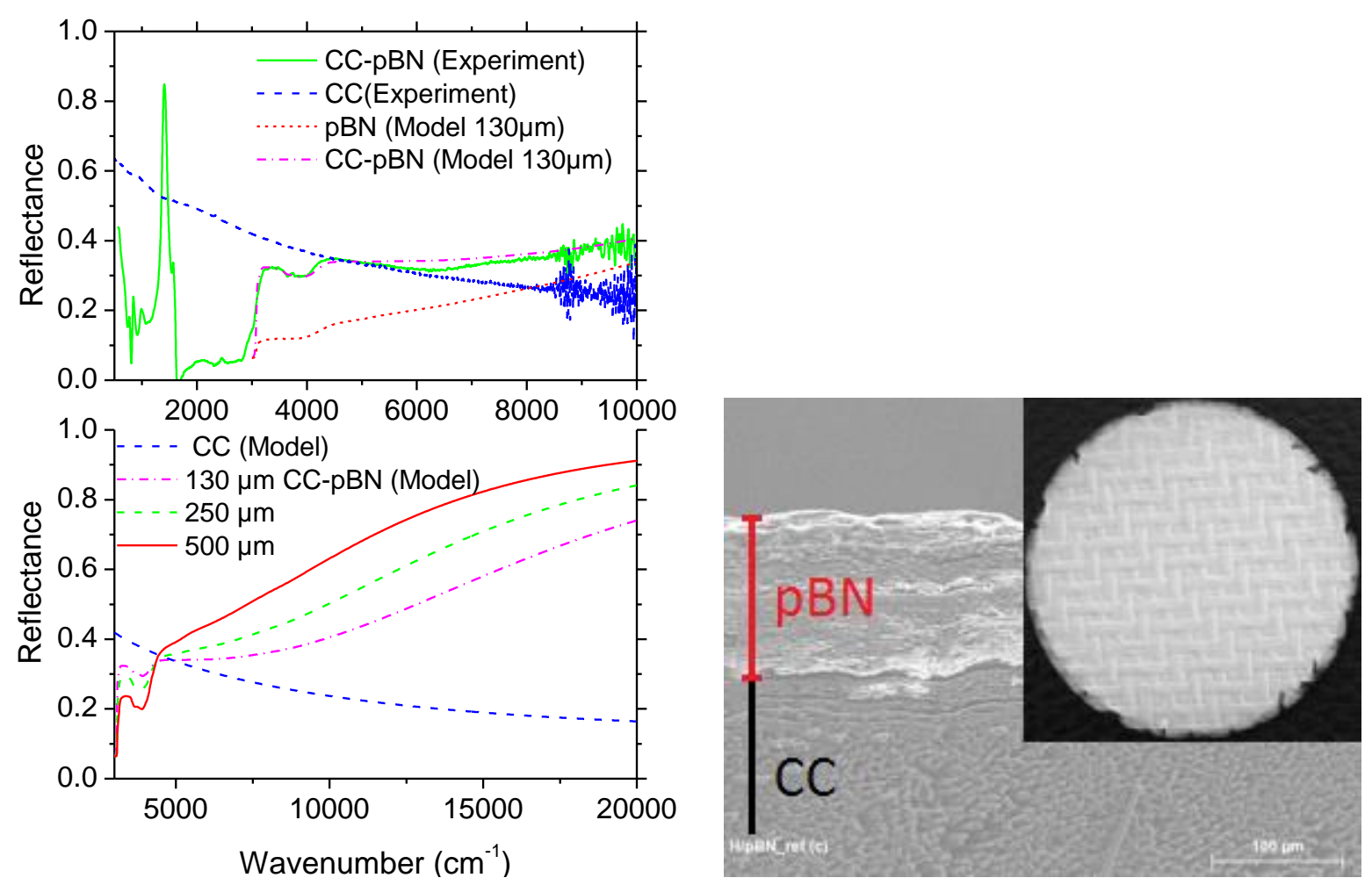

Fig. 7: Normal hemispherical reflectance spectra of carbon-carbon composite (CC) and of carbon-carbon composite coated with $130 \mu \mathrm{m}$ of $\mathrm{pBN}(\mathrm{CC}-\mathrm{pBN})$. Estimations of the reflectance of coated carbon-carbon composite with increasing thicknesses obtained with Eq. (10) are displayed in the lower part of the figure.

A way to improve the thermal radiation characteristics of shields built with $2.5 \mathrm{D}$ carboncarbon composites, while maintaining their excellent structural qualities, is to coat them with optically selective materials such as $\mathrm{pBN}$. $\mathrm{pBN}$ has the additional advantage of being highly compatible with carbon-carbon composites even at extreme temperatures [4,5]. The impact of a $130 \mu \mathrm{m}$ thick pBN coating deposited by the CVD technique on the carbon-carbon composite 
is shown in Fig. 7. The coating imposes its excellent emitting properties in the mid-infrared range and improves significantly the reflectance level in the near-infrared and visible ranges. An estimation of the capacity of the coated shield to reflect the solar radiation in the semitransparent region due to the deposition of $\mathrm{pBN}$ with a given thickness $L$ can be computed using the following approximate formula:

$$
R_{d-L}^{C C-p B N} \cong R_{d-L}^{p B N}+\left(T_{d-L}^{p B N}\right)^{2}\left(R_{C C}-R_{p B N}\right)\left(1-R_{p B N}\right)
$$

In this expression, $R_{d-L}^{p B N}$ and $T_{d-L}^{p B N}$ are the normal hemispherical reflectance and transmittance of an isolated layer of pBN having a thickness equal to $L . R_{C C}$ and $R_{p B N}$ are the interface reflectivities of the carbon-carbon composite and pBN, respectively. The results obtained by applying Eq. (10) for pBN thicknesses going from $130 \mu \mathrm{m}$ to $500 \mu \mathrm{m}$ are reported on the bottom of Fig. 7. Eqs. (6) and (7) have been used with the absorption and scattering coefficients extracted for the 1.5 mm thick pBN sample to evaluate the reflectance and transmittance of the coating versus thickness. Approximations of the interface reflectivities were computed with Eq. (4). The comparison between the reflectance $R_{d-130 \mu m}^{C C-p B N}$ computed with Eq. (10) and the experimental one shows that the approximation is reasonable (upper part of Fig. 7) and legitimates its use to estimate the optical response of carbon-carbon samples coated with other thicknesses of pBN. As shown on the lower part of Fig. 7 which includes the strongest part of the solar spectrum, the reflectance $R_{d-L}^{C C-p B N}$ of $\mathrm{pBN} /$ carbon-carbon composites is strongly improved in comparison to the one of bare carbon-carbon composite. A very high level of reflectance is achieved with a coating thickness of $500 \mu \mathrm{m}$.

\section{Conclusion}

Textured $\mathrm{pBN}$ materials have very specific properties that make them a must for a lot of applications. Their optical characterization is thus necessary since absorption and scattering 
coefficients are required to size systems such as thermal protections. This paper reports on the dielectric properties of highly oriented boron nitride and textured $\mathrm{pBN}$. The results confirm the strong optical anisotropy of boron nitride predicted by first principle calculations and validate the modified two flux model to extract accurate absorption and scattering properties of $\mathrm{pBN}$ in the mid-infrared range. The deposition of a layer of $\mathrm{pBN}$ on carbon-carbon composites strongly increases their protection performance for a use as solar heat shields. An approximate expression using the optical properties of $\mathrm{pBN}$ and of the carbon-carbon composite was given to predict the normal hemispherical reflectance of coated carbon-carbon composite samples versus the thickness of the pBN layer.

\section{Acknowledgement}

The authors are grateful to Emanuel Veron for performing X-ray measurements on the $\mathrm{pBN}$ sample.

\section{References}

1. Selvakumar N, Barshilia HC (2012) Review of physical vapor deposited (PVD) spectrally selective coatings for mid- and high-temperature solar thermal applications. Sol Energ Mat Sol C 98:1-23

2. Golosnoy I O, Cipitria A, Clyne T W (2009) Heat Transfer Through Plasma-Sprayed Thermal Barrier Coatings in Gas Turbines: A Review of Recent Work. J Therm Spray Technol 18:809-821

3. Wang L, Habibi MH, Eldridge JI et al (2014) Infrared radiative properties of plasmasprayed $\mathrm{BaZrO}_{3}$ coatings. J Eur Ceram Soc 34:3941-3949 
4. Balat-Pichelin M, Eck J, Heurtault S, Glénat H (2014) Experimental study of pyrolytic boron nitride at high temperature with and without proton and VUV irradiations. Appl Surf Sci. $314: 415-425$

5. Brodu E, Balat-Pichelin M, De Sousa Meneses D et al (2015) Reducing the temperature of a C/C composite heat shield for solar probe missions with an optically selective semitransparent pyrolytic boron nitride (pBN) coating. Carbon 82:39-50.

6. De Sousa Meneses D, Brun JF, Echegut P et al (2004) Contribution of semi-quantum dielectric function models to the analysis of infrared spectra. Appl Spectrosc 58:969-974 7. Sacadura J-F (2011) Thermal Radiative Properties of Complex Media: Theoretical Prediction Versus Experimental Identification. Heat Transfer Eng 32:754-770

8. Dombrovsky LA, Randrianalisoa JH, Lipinski W et al (2011) Approximate analytical solution to normal emittance of semi-transparent layer of an absorbing, scattering, and refracting medium. J Quant Spectrosc Radiat Transfer 112:1987-1994

9. Dombrovsky LA (2012) The use of transport approximation and diffusion-based models in radiative transfer calculations. Comput Therm Sci 4:297-315

10. Randrianalisoa J, Baillis D, Pilon (2006) Improved Inverse Method for Radiative Characteristics of Closed-Cell Absorbing Porous Media. J Thermophys Heat Transfer 20:871883

11. Dombrovsky LA, Tagne HK, Baillis D et al (2007) Near-infrared radiative properties of porous zirconia ceramics. Infrared Phys Technol. 51:44-53

12. Wang L, Eldridge JI, Guo SM (2014) Comparison of different models for the determination of the absorption and scattering coefficients of thermal barrier coatings. Acta Materialia 64:402-410

13 Geick R, Perry CH, Rupprecht G (1966) Normal Modes in Hexagonal Boron Nitride. Phys Rev 146:543-547 
14. Hoffman D M, Doll GL, Eklund PC (1984) Optical properties of pyrolytic boron nitride in the energy range 0.05- 10 eV. Phys Rev B 30:6051-6056

15. Schubert M, Rheinlander B, Franke E et al (1997) Infrared optical properties of mixedphase thin films studied by spectroscopic ellipsometry using boron nitride as an example. Phys Rev B 56:13306-13313

16. Ben el Mekki M, Mestres N, Pascual J et al (1999) Infrared and Raman analysis of plasma CVD boron nitride thin films. Diamond Relat Mater 8:398-401

17. Xu Y-N, Ching WY (1991) Calculation of ground-state and optical properties of boron nitrides in the hexagonal, cubic, and wurtzite structures. Phys Rev B 44:7787-7798

18 Ohba N, Miwa K, Nagasako N et al (2001) First-principles study on structural, dielectric, and dynamical properties for three BN polytypes. Phys Rev B 63:115207-9

19. Serrano J, Bosak A, Arenal R et al Vibrational (2007) Properties of Hexagonal Boron Nitride: Inelastic X-Ray Scattering and Ab Initio Calculations. Phys Rev Lett 98:095503-4 20. Yu WJ, Lau WM, Chan SP, Liu ZF et al (2003) Ab initio study of phase transformations in boron nitride. Phys Rev B 67:014108-9

21. Cai Y, Zhang L, Zeng Q, Cheng L, Xu Y (2007) Infrared reflectance spectrum of BN calculated from first principles. Solid State Communications 141:262-266

22. Hamdi I, Meskini N (2010) Ab initio study of the structural, elastic, vibrational and thermodynamic properties of the hexagonal boron nitride: Performance of LDA and GGA, Physica B 405:2785-2794

23. Manara J, Caps R, Ebert H-P et al (2002) Infrared optical properties of semitransparent pyrolytic boron nitride (pBN). High Temp High Press 34:65-72

24. Manara J, Keller M, Kraus D et al (2008) Determining the transmittance and emittance of transparent and semitransparent materials at elevated temperatures. In: Proceedings of the 5th European Thermal-Sciences Conference, The Netherlands 
25. Moore AW (1969) Compression Annealing of Pyrolytic Boron Nitride. Nature 221:11331134

26. Dupleix A, De Sousa Meneses D, Hughes M et al (2013) Mid-infrared absorption properties of green wood, Wood Science and Technology 47:1231-1241 27 Pease RS (1952) An X-ray study of boron nitride. Acta Crystallogr 5:356-361 28. Zhang D, Cherkaev E, Lamoureux MP (2011) Stieltjes representation of the 3D Bruggeman effective medium and Padé approximation. Appl Math Comput 217:7092-7107 29. Andersson SK, Ribbing CG (1994) Light extinction by bulk voids and surface irregularities in ceramic materials. Phys Rev B 49:11336-11343 30. Nemanich RJ, Solin SA, Martin RM (1981) Light scattering study of boron nitride microcrystals. Phys Rev B 23:6348-6356 